\title{
Statistical Evaluation of New Estimators used in Forced Oscillation Source Localization
}

\author{
Jim Follum \\ Pacific Northwest National Laboratory \\ james.follum@pnnl.gov
}

\begin{abstract}
Forced oscillations occur in power systems when a piece of equipment injects a periodic disturbance into the system. Under certain conditions, these oscillations can become large and widespread, impacting the system's reliability. Several methods have been proposed to identify the oscillation's source so that it can be addressed. One of the most promising is based on Dissipating Energy Flow (DEF). In this paper, a new expression for the DEF is developed under a set of commonly observed conditions. Based on this new expression, three new DEF estimators are proposed. The performance and sensitivities of these estimators, along with a previously published method, are evaluated using statistical methods. Results from simulated and measured power system data validate the DEF estimators and highlight the advantages and disadvantages of each.
\end{abstract}

\section{Introduction}

The development of extensive phasor measurement unit (PMU) networks has led to a vastly improved wide-area view of power systems. One application that has drawn considerable attention in recent years is the identification and mitigation of forced oscillations (FOs). Forced oscillations occur when the system is subjected to a periodic input, resulting in swings in voltage, frequency, and power. These inputs vary widely and can be caused by equipment failures, malfunctioning control systems, or abnormal operating conditions [1, 2]. Representative examples include a misoperating turbine governor [3], a hydro unit undergoing vortex oscillations while operating in the rough zone [4], and a malfunctioning steam extractor control valve [5]. Forced oscillations have also been

The Pacific Northwest National Laboratory is operated for the U.S. Department of Energy by Battelle Memorial Institute under Contract DE-AC05-76RL01830. This work was funded by the U.S. DOE. found to originate from oscillating loads [3] and inverter-based generation sources [6].

As noted in [3] and [1], FOs occur regularly in power systems. Most events do not pose a significant threat to grid reliability. However, system-wide high-energy oscillations do occur, particularly when the FO frequency aligns with the frequency of a natural system mode of oscillation $[1,3,4,5]$. Even if a FO is not a threat to reliability, it may limit system operation and cause additional wear on equipment. For these reasons, it is important that utilities are able to quickly identify an oscillation's source.

Identifying the source of a FO is a challenging problem. Due to the interaction between the oscillation and the system's dynamics, FOs are not always largest near their source. Even methods using a model to account for the system's dynamics may fail under certain conditions [7]. Additionally, the wide variety in equipment, control systems, and operating conditions that can cause a FO make it difficult to develop a method general enough to be applied in all situations. Despite these difficulties, many methods based on PMU measurements have been proposed. A survey of localization methods is presented in [2]. The authors classify methods into four major categories: damping torque, mode shape estimation, traveling wave theory, and energy. An energy-based method of particular promise is the focus of this paper.

The Dissipating Energy Flow (DEF) method calculates the flow of dissipating transient energy through the network using PMU measurements [8]. It is shown in [8] that system components producing energy are the oscillation sources. In [1], the method is refined for practical use by band-pass filtering measurements at the frequency of the oscillation. The authors of [1] also note the superior reliability of the DEF method in comparison with alternatives based on evaluation with a test case library [9]. The method was transformed to the frequency domain and extended to monitor a system component's damping contribution using ambient measurements in [10]. 
This paper makes several contributions to the study of the DEF method. First, a new expression for the DEF under certain common conditions is derived. Based on this expression, three novel methods to estimate the DEF are proposed. The advantages and disadvantages of these methods are evaluated alongside the approach in [1]. Where previous papers have evaluated methods based on a limited set of test cases, the results presented here go further by evaluating the statistical performance of DEF estimators using simulated cases. The methods are also evaluated using measured data to show their practicality under real-world conditions. The methods and results presented in this paper will help the DEF method be applied in the most effective possible way.

The rest of the paper is organized as follows. In Section 2, the new expression for the DEF is derived. Methods for evaluating the expression are proposed in Section 3 and then evaluated in Section 4. A discussion of results is provided in Section 5, followed by conclusions in Section 6 .

\section{A New Expression for Dissipating Energy Flow}

In this section, a new expression for the DEF is derived. The derivation begins from the expression for DEF provided in [8], which depends on measurements of active power, reactive power, system frequency, and voltage magnitude. Denote the measurements of active power as

$$
P(t)=P_{s}+P_{f}(t)
$$

where $P_{s}$ is the steady-state term and the FO term is

$$
P_{f}(t)=A_{P} \cos \left(\omega_{F O} t+\theta_{P}\right)
$$

where $A_{P}, \omega_{F O}$, and $\theta_{P}$ are the amplitude, frequency in radians per second, and phase of the FO. Additionally, let $Q(t)=Q_{s}+Q_{f}(t), \Omega(t)=\Omega_{s}+\Omega_{f}(t)$, and $V(t)=V_{s}+V_{f}(t)$ denote measurements of reactive power, system frequency, and voltage magnitude. The FO components of these measurements have the same form as (2). The angular frequency $\Omega(t)$ arises in the derivation for DEF in [8] as the derivative of voltage angle. In this paper, the scaled first-order difference of the measured voltage angle is used to obtain the "measurement" of $\Omega(t)$. This approach is commonly used to avoid the variation in frequency measurements between different PMU manufacturers. To simplify notation, the dependence on time of FO components will be omitted for the remainder of the paper, i.e., $P_{f}(t)=P_{f}$.

As the result of periodic disturbances, FOs can typically be well represented as a sum of sinusoids at harmonic frequencies [11, 12]. For the initial development, a single sinusoid is considered. As discussed later, practical implementation of the derived algorithms addresses these harmonics so that the theory developed here holds.

As detailed in $[1,8,10]$, the DEF can be expressed in terms of measured power system quantities as

$$
W=\int P_{f} d\left(\theta_{f}\right)+\int Q_{f} d\left(U_{f}\right)
$$

where $\theta_{f}$ denotes the FO component of the voltage angle, and $U_{f}$ denotes the FO component of $U(t)=$ $\ln (V(t))$. Because $P_{f}, \theta_{f}, Q_{f}$, and $U_{f}$ are all functions of time, the integrals can be rewritten as

$$
W=\int_{0}^{T} P_{f} \Omega_{f} d t+\int_{0}^{T} Q_{f} U_{f}^{\prime} d t
$$

where $T$ is the analysis length in seconds, which is selected to contain an integer number of FO cycles, and $'$ indicates the first derivative. To obtain an expression for $U_{f}^{\prime}$, note that

$$
\begin{aligned}
U(t) & =\ln \left(V_{s}+A_{V} \cos \left(\omega_{F O} t+\theta_{V}\right)\right) \\
& \approx \ln \left(V_{s}\right)+\frac{A_{V}}{V_{s}} \cos \left(\omega_{F O} t+\theta_{V}\right)
\end{aligned}
$$

for $V_{s} \gg A_{V}$ (see the appendix for further discussion). Letting $U_{s}=\ln \left(V_{s}\right), A_{U}=\frac{A_{V}}{V_{s}}$, and $\theta_{U}=\theta_{V}$,

$$
\begin{aligned}
U(t) & =U_{s}+A_{U} \cos \left(\omega_{F O} t+\theta_{U}\right) \\
& =U_{s}+U_{f}(t)
\end{aligned}
$$

The derivative of the FO component follows as

$$
U_{f}^{\prime}=-\omega_{F O} A_{U} \sin \left(\omega_{F O} t+\theta_{U}\right) .
$$

With each term in (4) expressed as a sinusoid, the expression for the DEF can be greatly simplified. Applying the trigonometric identity

$$
\cos (\alpha) \cos (\beta)=\frac{1}{2} \cos (\alpha-\beta)+\frac{1}{2} \cos (\alpha+\beta),
$$

the first integral can be written as

$$
\begin{aligned}
\int_{0}^{T} P_{f} \Omega_{f} d t= & \frac{1}{2} A_{P} A_{\Omega} \int_{0}^{T}\left[\cos \left(\theta_{P}-\theta_{\Omega}\right)\right. \\
& \left.+\cos \left(2 \omega_{F O} t+\theta_{P}+\theta_{\Omega}\right)\right] d t
\end{aligned}
$$

Noting that the integral of a sinusoid over an integer number of periods is equal to zero,

$$
\int_{0}^{T} P_{f} \Omega_{f} d t=\frac{T}{2} A_{P} A_{\Omega} \cos \left(\theta_{P}-\theta_{\Omega}\right) .
$$


Similarly, applying the trigonometric identity

$$
\cos (\alpha) \sin (\beta)=\frac{1}{2} \sin (\alpha-\beta)+\frac{1}{2} \sin (\alpha+\beta)
$$

allows the second integral in (4) to be expressed as

$$
\int_{0}^{T} Q_{f} U_{f}^{\prime} d t=\frac{T}{2} A_{Q} A_{U} \omega_{F O} \sin \left(\theta_{Q}-\theta_{U}\right) .
$$

Combining the results in (10) and (12) leads to the final expression

$$
\begin{aligned}
W=\frac{T}{2}\left[A_{P} A_{\Omega}\right. & \cos \left(\theta_{P}-\theta_{\Omega}\right) \\
& \left.+A_{Q} A_{U} \omega_{F O} \sin \left(\theta_{Q}-\theta_{U}\right)\right]
\end{aligned}
$$

\section{Evaluating the New Expression}

The expression for DEF in (13) is general in that there are multiple ways for it to be evaluated using synchronized measurements. Three approaches are explored in this paper.

Though the DEF is expressed in terms of continuous time in the previous section, the PMU data used to estimate it is sampled at a regular interval. Thus, the methods discussed in this section are derived using discrete time notation. Let $k=0,1, \ldots, K-1$ denote the sample indices within the analysis window corresponding to a sampling period $T_{s}$ such that $t=$ $k \times T_{s}$. Then the term $\omega_{F O}^{k}=\omega_{F O} \times T_{s}$ denotes the FO's frequency in radians per sample.

\subsection{DFT Approach}

In the first approach, the amplitudes and phases in (13) are estimated based on the Discrete Fourier Transform (DFT) of the measurements. Begin by noting that

$$
\left.\operatorname{DFT}\left\{P(k)-P_{\mu}\right\}\right|_{\omega^{k}=\omega_{F O}^{k}} \approx \hat{P}_{f}\left(\omega_{F O}^{k}\right)
$$

where $P_{\mu}$ denotes the average of $P(k)$ over the analysis window and $\hat{P}_{f}\left(\omega^{k}\right)$ denotes the DFT of $P_{f}(k)$. Because $P_{f}(k)$ is a cosine, it follows that

$$
\hat{P}_{f}\left(\omega_{F O}^{k}\right)=\frac{K}{2} A_{P} \angle \theta_{P}
$$

Similar relationships hold for the other measured quantities. Thus, amplitude and phase estimates can be obtained by removing the mean from each set of measurements, calculating the DFT, and finding the magnitude and phase at the frequency of the FO. These estimates are then plugged into (13) to estimate the DEF.

\subsection{Cross-Spectrum Approach}

The second approach also leverages the calculation of DFTs for each type of measurement. In fact, the resulting DEF estimates are identical to those in the first method. However, the amplitude and phase for each measurement are not directly estimated. The approach is included here because it links the results derived in the previous section to another published method.

To begin, consider the cross-power spectral density (CPSD) [13] evaluated at the FO frequency:

$$
\begin{aligned}
S_{P \Omega}\left(\omega_{F O}^{k}\right) & =\frac{1}{K} \hat{P}_{f}\left(\omega_{F O}^{k}\right) \hat{\Omega}_{f}^{*}\left(\omega_{F O}^{k}\right) \\
& =\frac{K}{4} A_{P} A_{\Omega} \angle\left(\theta_{P}-\theta_{\Omega}\right) \\
& =\frac{K}{4} A_{P} A_{\Omega}\left[\cos \left(\theta_{P}-\theta_{\Omega}\right)\right. \\
& \left.\quad+j \sin \left(\theta_{P}-\theta_{\Omega}\right)\right]
\end{aligned}
$$

Here ${ }^{*}$ denotes the complex conjugate and $j=\sqrt{-1}$. Similarly,

$S_{Q U}\left(\omega_{F O}^{k}\right)=\frac{K}{4} A_{Q} A_{U}\left[\cos \left(\theta_{Q}-\theta_{U}\right)+j \sin \left(\theta_{Q}-\theta_{U}\right)\right]$.

Comparing (16) and (17) with (13), the CPSD-based estimator can be expressed as

$$
W=2 T_{s}\left[\operatorname{Re}\left\{S_{P \Omega}\left(\omega_{F O}^{k}\right)\right\}+\omega_{F O} \operatorname{Im}\left\{S_{Q U}\left(\omega_{F O}^{k}\right)\right\}\right]
$$

where $\operatorname{Re}\{\cdot\}$ and $\operatorname{Im}\{\cdot\}$ are functions returning real and imaginary components, respectively.

The CPSDs in this expression can be estimated using the DFTs of measured data as in (14). While successful spectral analysis of stochastic signals typically requires long record lengths, non-rectangular windows, and averaging of multiple overlapping windows, the same is not true for the deterministic periodic signals considered here [13]. This greatly simplifies the design of the DFTand CPSD-based DEF estimators.

The result in (18) is closely related to the method in [10], where an expression for the damping-torque coefficient is derived that contains the following frequency-domain formulation of the DEF:

$$
W=2 T_{s} \operatorname{Re}\left\{S_{P \Omega}\left(\omega_{F O}^{k}\right)+S_{Q U}\left(\omega_{F O}^{k}\right)\right\} .
$$

This expression was also derived based on (4), though a very different approach was taken. The paper focuses on ambient measurements and does not mention FOs. The similarities between (18) and (19) warrant discussion and further investigation. However, because the current paper focuses specifically on DEF estimation for FOs, the estimator in (19) is not evaluated. 


\subsection{Least-Squares Approach}

The final approach to estimating the DEF is also based on estimating the amplitudes and phases of the FO in each measurement, but it operates in the time domain. The two previously proposed methods operate in the frequency domain and ignore additional signal components and harmonics by only analyzing the frequency bin corresponding to the the FO. In [1], these additional components were removed by applying a band-pass filter centered at the FO's frequency. The method proposed in this section accounts for non-FO signal components by fitting the entire input signal to a model.

The approach is based on the Least-Squares plus Sinusoid (LS+S) method for estimating a system's electromechanical modes in the presence of FOs [14]. To do this, it incorporates a FO and its harmonics into the system model. A least-squares (LS) approach is used to estimate the system parameters and the FO amplitudes and phases simultaneously. In the present application, accurate estimation of the system's dynamics, which would require additional signal processing and careful parameter selection, is not of interest. Thus, the system model is estimated only to provide more accurate estimation of the FO parameters.

In the following, an overview of the first stage of the $\mathrm{LS}+\mathrm{S}$ algorithm is provided. The second stage is similar and updates the estimates from the first stage. See [14] for a complete description. Equations are provided for active power measurements, but the same approach is applied for the other measurements. For simplicity, only a single oscillation frequency will be considered here. As described in [14], the method can readily be extended for multiple oscillations.

To begin, redefine the active power measurements as

$$
P(k)=P_{s}+P_{n}(k)+P_{f}(k)
$$

where $P_{n}(k)$ is a stochastic component due to random changes, e.g., load, that excite the system's dynamics. This component has been ignored up to this point because frequency domain techniques [10] and band-pass filtering [1] largely remove its impact. Next, let

$$
P_{0}(k)=P_{s}+P_{n}(k)+P_{f}(k)-P_{\mu} \approx P_{n}(k)+P_{f}(k)
$$

The noise component can be modeled as an auto-regressive (AR) process, resulting in the expression

$$
P_{n}(k)=-\sum_{i=1}^{n_{a}} a_{i} P_{n}(k-i)+e(k)
$$

where $e(k)$ represents the noise driving the system's dynamics. Applying trigonometric identities and writing the equation for $P_{0}(k)$ out for $n_{a} \leq k \leq K-1$ leads to the matrix equation

$$
\bar{P}_{0}=\left[\begin{array}{ll}
\boldsymbol{P}_{\mathbf{0}} & \boldsymbol{F}
\end{array}\right] \bar{\psi}
$$

where

$$
\begin{aligned}
& \bar{P}_{0}=\left[\begin{array}{llll}
P_{0}\left(n_{a}\right) & P_{0}\left(n_{a}+1\right) & \cdots & P_{0}(K-1)
\end{array}\right]^{T} \\
& \boldsymbol{P}_{\mathbf{0}}=\left[\begin{array}{ccc}
P_{0}\left(n_{a}-1\right) & \cdots & P_{0}(0) \\
P_{0}\left(n_{a}\right) & \cdots & P_{0}(1) \\
\vdots & & \vdots \\
P_{0}(K-2) & \cdots & P_{0}\left(K-n_{a}-1\right)
\end{array}\right] \\
& \boldsymbol{F}=\left[\begin{array}{cc}
\cos \left(\omega_{F O}^{k}\left(n_{a}\right)\right) & -\sin \left(\omega_{F O}^{k}\left(n_{a}\right)\right) \\
\cos \left(\omega_{F O}^{k}\left(n_{a}+1\right)\right) & -\sin \left(\omega_{F O}^{k}\left(n_{a}+1\right)\right) \\
\vdots & \vdots \\
\cos \left(\omega_{F O}^{k}(K-1)\right) & -\sin \left(\omega_{F O}^{k}(K-1)\right)
\end{array}\right] \\
& \bar{\psi}=\left[\begin{array}{lllll}
a_{1} & \cdots & a_{n_{a}} & B & C
\end{array}\right]^{T} .
\end{aligned}
$$

An estimate of the parameter vector can be obtained through the least-squares solution to (23) as

$$
\hat{\bar{\psi}}=\left[\begin{array}{ll}
\boldsymbol{P}_{\mathbf{0}} & \boldsymbol{F}
\end{array}\right]^{\dagger} \bar{P}_{0}
$$

where $\dagger$ indicates the pseudoinverse. The amplitude and phase of the FO can then be estimated as

$$
\hat{A}_{P}=\left|\frac{1}{\hat{A}\left(\omega_{F O}^{k}\right)}\right| \sqrt{\hat{B}^{2}+\hat{C}^{2}}
$$

$$
\hat{\theta}_{P}=\tan ^{-1}\left(\frac{\hat{C}}{\hat{B}}\right)+\angle \frac{1}{\hat{A}\left(\omega_{F O}^{k}\right)}
$$

where parameter estimates are indicated with hats and

$$
\begin{aligned}
\hat{A}\left(\omega_{F O}^{k}\right)=1+ & \hat{a}_{1} e^{-j \omega_{F O}^{k}} \\
& +\hat{a}_{2} e^{-j 2 \omega_{F O}^{k}}+\cdots+\hat{a}_{n_{a}} e^{-j n_{a} \omega_{F O}^{k}}
\end{aligned}
$$

is related to the transfer function of the AR model.

Though the LS+S method may appear complex, in practice it is relatively straightforward to implement. Measurements are first collected into the matrix $\boldsymbol{P}_{\mathbf{0}}$ and vector $\bar{P}_{0}$. Next, the $\boldsymbol{F}$ matrix is formed based on the frequency of the observed oscillation(s). An estimate of the parameter vector is calculated using (28), which can 
be implemented easily using a pseudoinverse function, such as MATLAB's pinv. Using the resulting parameter estimates, (29) and (30) are used to estimate the FO's amplitude and phase. The process is repeated for each set of measurements. Finally, (13) is used to estimate the DEF for each branch.

\section{Experimental Results}

To evaluate the proposed methods, they were applied to simulated and measured PMU data during FO conditions. Application to measured data helps ensure that an approach is practical for deployment under real-world conditions. Simulated data was used to perform statistical evaluations of DEF estimator performance using repeated trials. To the author's knowledge, this is the first publication to evaluate DEF estimators in this way. The simulated data allows evaluation of the methods' sensitivities to variations in input parameters. Together, the measured and simulated results provide a good indication of each method's performance and viability for practical deployment.

The evaluated methods include the "Direct" evaluation of (3) suggested in [1], the CPSD approach proposed in Section 3.2, and the LS method proposed in Section 3.3. Recall that the DFT method in Section 3.1 is mathematically equivalent to the CPSD approach. Results are presented in the following subsections with additional discussion provided in Section 5.

\subsection{Results from Simulated Data}

Simulated data was generated using the miniWECC model, a complete description of which is presented in [15] and the references therein. The miniWECC is a simplified model of the Western Electricity Coordinating Council (WECC) system obtained by equivalencing generation for many areas into single generators and including only transmission lines with significant length and a rating of at least $230 \mathrm{kV}$. In all, the model has 34 generators, 115 lines and high-voltage transformers, 54 generator and load transformers, 19 load buses, and 2 DC lines. For data generation, the nonlinear model is linearized about an operating point and represented in state-space form. The model has dominant electromechanical modes at $0.22 \mathrm{~Hz}$ with $7 \%$ damping, $0.37 \mathrm{~Hz}$ with $5 \%$ damping, $0.62 \mathrm{~Hz}$ with $6.2 \%$ damping, and $0.73 \mathrm{~Hz}$ with $6.6 \%$ damping. The relatively low damping ratios of the modes increases the system's susceptibility to FOs appearing system wide [4].

Forced oscillations are modeled as a square wave input at a generator bus to emulate a limit cycle [16]. The square wave amplitude is specified in terms of the
Table 1. Simulation cases.

\begin{tabular}{||cccc||}
\hline Case & Freq. (Hz) & Amp. (pu) & Input Gen. \\
\hline \hline 1 & 0.37 & 0.005 & 1 \\
\hline 2 & 0.37 & 0.005 & 7 \\
\hline 3 & 0.37 & 0.005 & 14 \\
\hline 4 & 0.37 & 0.005 & 21 \\
\hline 5 & 0.37 & 0.005 & 30 \\
\hline \hline 6 & 0.22 & 0.001 & 1 \\
\hline 7 & 0.22 & 0.001 & 7 \\
\hline 8 & 0.22 & 0.001 & 14 \\
\hline 9 & 0.22 & 0.001 & 21 \\
\hline 10 & 0.22 & 0.001 & 30 \\
\hline
\end{tabular}

generator's input mechanical power. Two sets of cases are considered, each with a frequency corresponding to an electromechanical mode. In the "large FO" cases, a $0.37 \mathrm{~Hz}$ square wave with an amplitude of 0.005 per unit is applied. In the "small FO" cases, the oscillation's fundamental frequency was set to $0.22 \mathrm{~Hz}$ and its amplitude was set to 0.001 per unit. For each set of cases, the FO was injected at the five generators indicated in Fig. 1, leading to the ten cases listed in Table 1. The DEF was estimated at each of the model's 34 generators. Examples of the model outputs for two cases are presented in Fig. 2. The FO was injected beginning at the $60^{\text {th }}$ second of 180 -second simulations.

For each of the 10 cases, 100 trials were generated, each with a unique realization of the load noise driving the system and the measurement noise added to the model's output. Calculating the mean, standard deviation, and root mean squared error (RMSE) of the estimates over these trials provides an indication of each algorithm's statistical performance. This approach is known as a Monte Carlo simulation [17]. For the purposes of this study, the true DEF was calculated using (3) applied to sinusoids with amplitudes and phases calculated based on the transfer functions of the state space matrices composing the miniWECC model. Of course, the ultimate goal of DEF-based algorithms is to correctly identify the source of the FO. The impact of inaccurate DEF estimation on source localization is reported in terms of the percentage of trials where the source generator was accurately identified.

4.1.1. Base Case Performance To begin, each estimator was evaluated under ideal circumstances. The FO's true frequency was utilized and the analysis window was selected such that the FO amplitude remained relatively constant. The analysis window was approximately 60 seconds long and adjusted to contain 


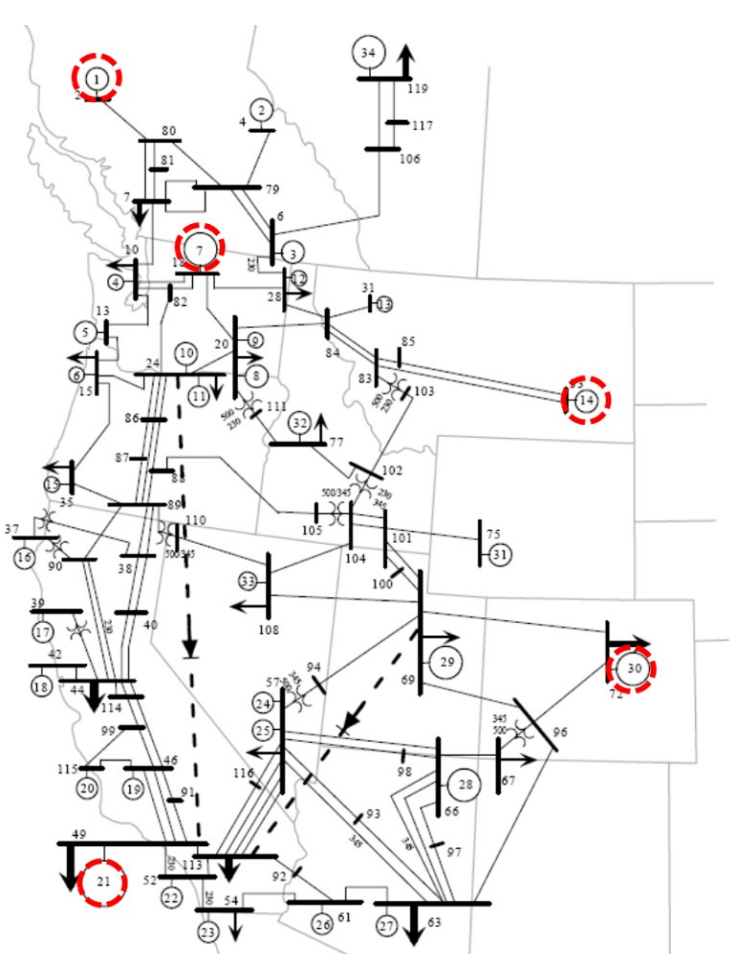

Figure 1. MiniWECC model indicating generators used to inject FOs. From top to bottom: 1, 7, 14, 30,

21.
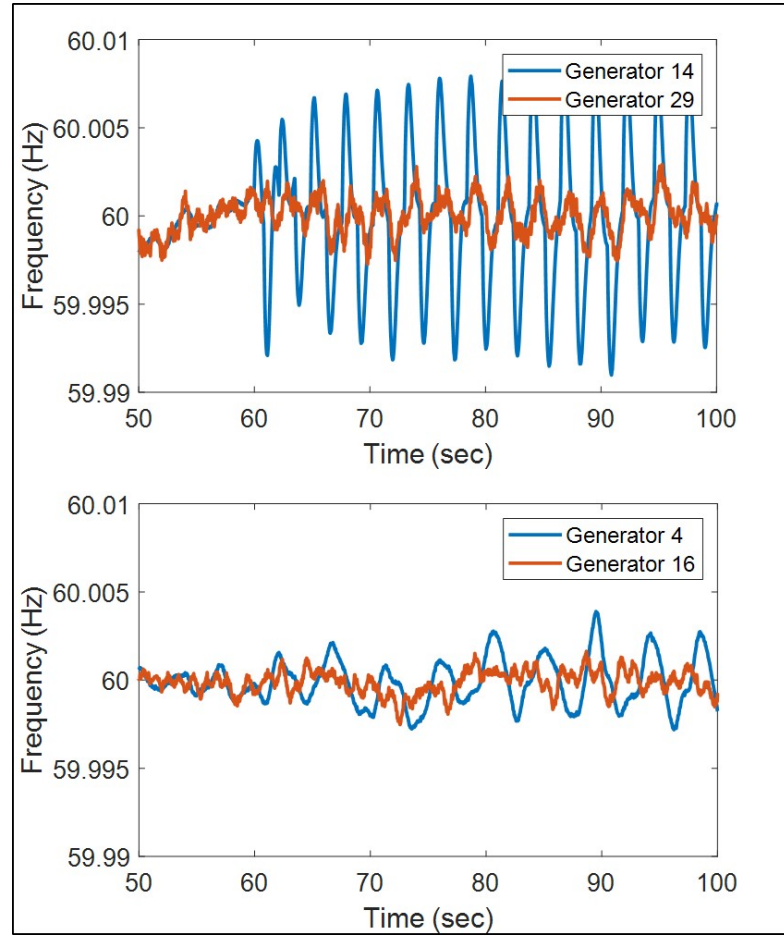

Figure 2. Example simulation data from cases 1 (top) and 8 (bottom).

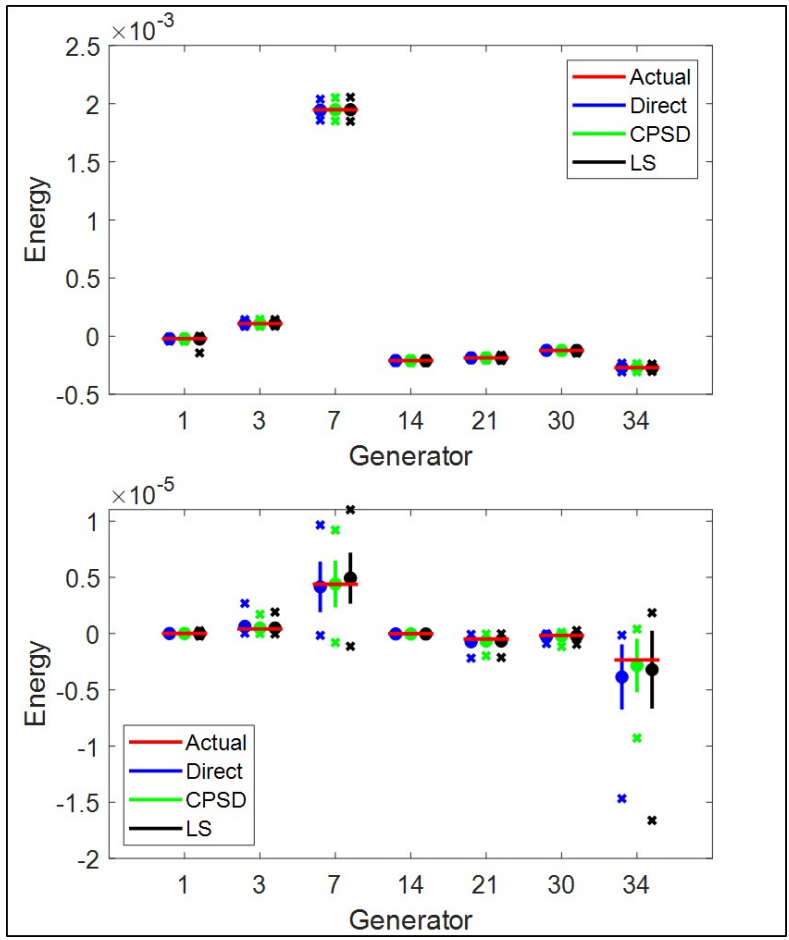

Figure 3. Estimation performance for case 2 (top) and 7 (bottom). The mean of estimates across trials are denoted with dots; vertical lines indicate standard

deviation above/below the mean, and $\max / \mathrm{min}$ estimates are marked with $x$.

an integer number of $\mathrm{FO}$ periods.

Results from cases 2 and 7 are plotted in Fig. 3 for a subset of generators. These plots reflect typical performance observed in the ten base cases. Considering the results from all ten cases, the three algorithms were found to provide very similar DEF estimation performance. As expected, the bias and variance become less significant in cases 1-5, where the FO amplitude is larger, because the signal-to-noise ratio (SNR) is higher. The percent of trials where the oscillation source was correctly identified are listed in Table 2. Again, the algorithms perform similarly, except for a slight improvement by the CPSD method in case 8 .

Given the similar performance among estimators in the base cases, their sensitivities to variations in input parameters are important for selecting a method for practical deployment. These sensitivities are evaluated in the following sections.

4.1.2. Sensitivity to Frequency Errors To evaluate the sensitivity of the DEF estimators to errors in FO frequency estimates, the RMSE was calculated for 
Table 2. Percent of trials with correctly identified source in base cases.

\begin{tabular}{||cccc||}
\hline Case & Direct & CPSD & LS \\
\hline \hline 1 & 100 & 100 & 100 \\
\hline 2 & 100 & 100 & 100 \\
\hline 3 & 100 & 100 & 100 \\
\hline 4 & 100 & 100 & 100 \\
\hline 5 & 100 & 100 & 100 \\
\hline \hline 6 & 99 & 99 & 97 \\
\hline 7 & 98 & 99 & 97 \\
\hline 8 & 65 & 72 & 63 \\
\hline 9 & 100 & 100 & 100 \\
\hline 10 & 100 & 100 & 100 \\
\hline
\end{tabular}

errors up to $1 / 60 \mathrm{~Hz}$. This range corresponds to one frequency bin of a DFT calculated using 60 seconds of data. DEF estimation performance in terms of RMSE is plotted for cases 4 and 9 in Fig. 4 for generator 21, the oscillation's source. Performance was similar for other cases and at other generators. The Direct method significantly outperforms the CPSD and LS methods. As suggested in [1], a bandpass filter centered at the FO frequency is applied as part of the Direct method. The width of the passband makes the method robust to errors in the FO frequency. The sensitivity of the LS method to frequency errors was noted in [14].

The impact of DEF estimation errors are reflected in the source localization results plotted in Fig. 5. Though the RMSE was similar for the CPSD and LS methods, the CPSD approach significantly outperforms the LS algorithm in identifying the oscillation's source. Interestingly, the LS method's localization performance degrades for the larger FO (case 4).

4.1.3. Sensitivity to Oscillation's Period In this section, the impact of analyzing a non-integer number of oscillation periods is evaluated. To conduct the study, the length of the analysis window was reduced in several steps to remove half a period of the analyzed FO. The results for case 5 are presented in Fig. 6 in terms of the RMSE at the source, generator 30. This is the most extreme case, but results consistently showed the CPSD method impacted in a similar manner. The increase in RMSE occurs due to leakage. When the DFT is applied to a non-integer number of cycles of a sinusoid, the frequency content "leaks" into nearby frequency bins, causing bias [18]. The other methods showed minimal impact. There was no appreciable change in the RMSE for the small FO cases 6-10. Additionally, there was no consistent impact to the source localization performance of any algorithm.

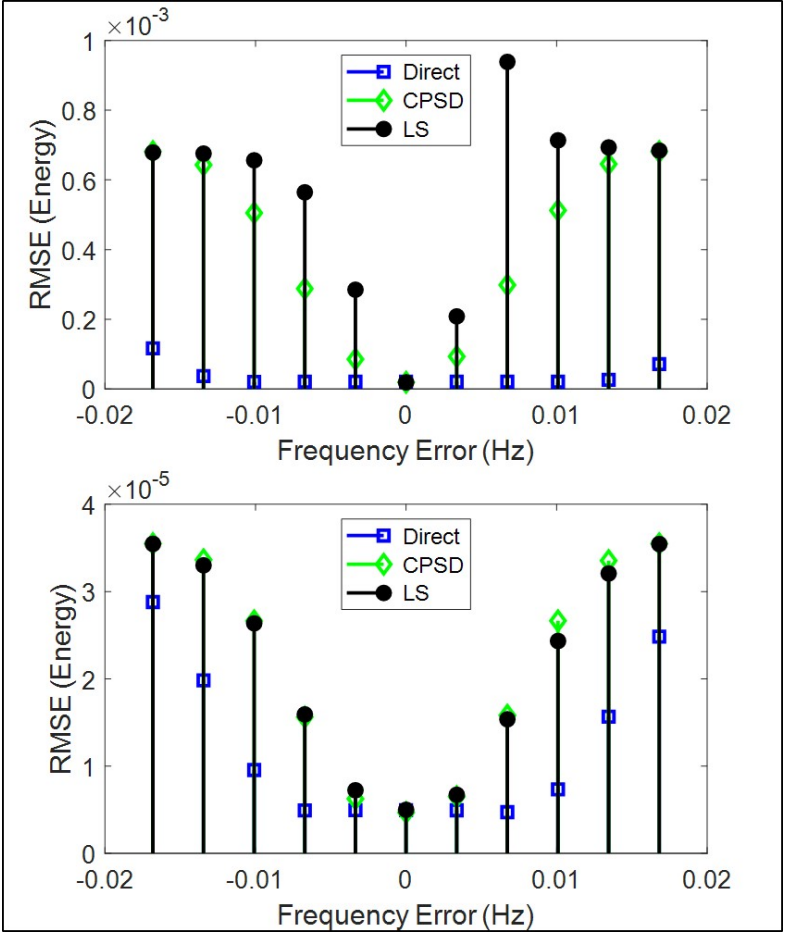

Figure 4. Estimation performance at the source generator for case 4 (top) and 9 (bottom) with frequency errors.
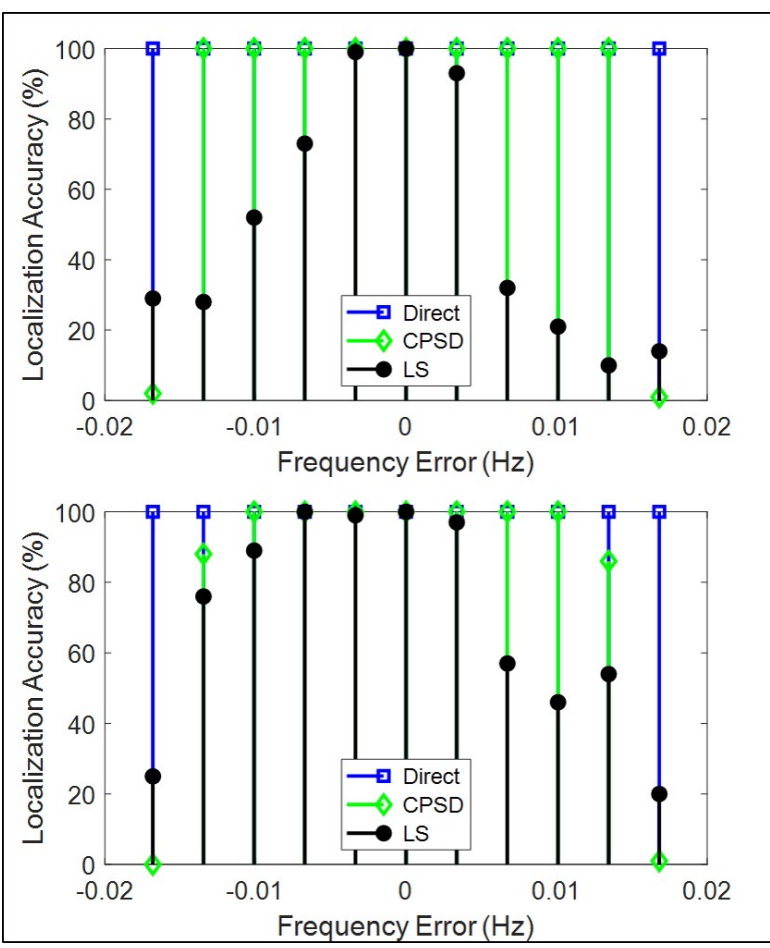

Figure 5. Source localization performance for case 4 (top) and 9 (bottom) with frequency errors. 


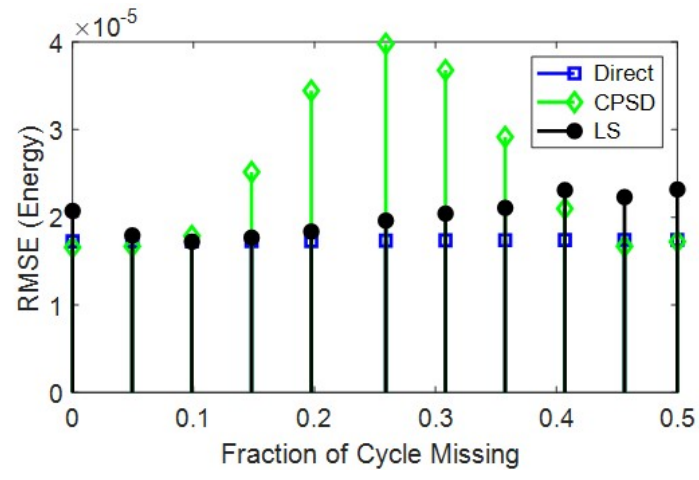

Figure 6. Estimation performance at the source generator for case 5 with a non-integer number of cycles.

4.1.4. Sensitivity to Window Selection As noted in [1], DEF estimation methods should be applied to analysis windows containing sustained oscillations. They do not elaborate on how to automatically identify the analysis window, but an algorithm for estimating the start and end points of a FO is proposed in [19]. In this section, the impact of errors in the analysis window selection is evaluated. The FOs in this study were initiated at the $60^{\text {th }}$ second of 180 -second simulations. For all results so far, the analysis window ran approximately from seconds $120-180$, when the FO is sustained. To test the sensitivity to window selection, the approximately 60 -second analysis window was shifted earlier in the simulation in several steps. For a shift of 90 seconds, the analysis window ran from $30-90$ seconds and contained only 30 seconds of the FO. Typical results are presented in Figs. 7 and 8.

\subsection{Results from Measured Data}

Tests on measurement data were conducted using publicly available datasets [9]. The Direct, CPSD, and LS estimators of the DEF successfully identified the oscillation source in each of the five available datasets. Here, the results from analysis of test case three are described in detail as an example.

As detailed in [9], the $1.13 \mathrm{~Hz}$ oscillation was caused by equipment malfunction in a large generator within the Independent System Operator of New England (ISO-NE) system. The oscillation persists for 40 seconds within the three-minute dataset. Thirty seconds of data was used to obtain the results presented here. A subset of the frequency measurements from the substation nearest to the oscillation source are presented in Fig. 9. Results of the DEF estimation are presented in Fig. 10. The methods were applied to each of the

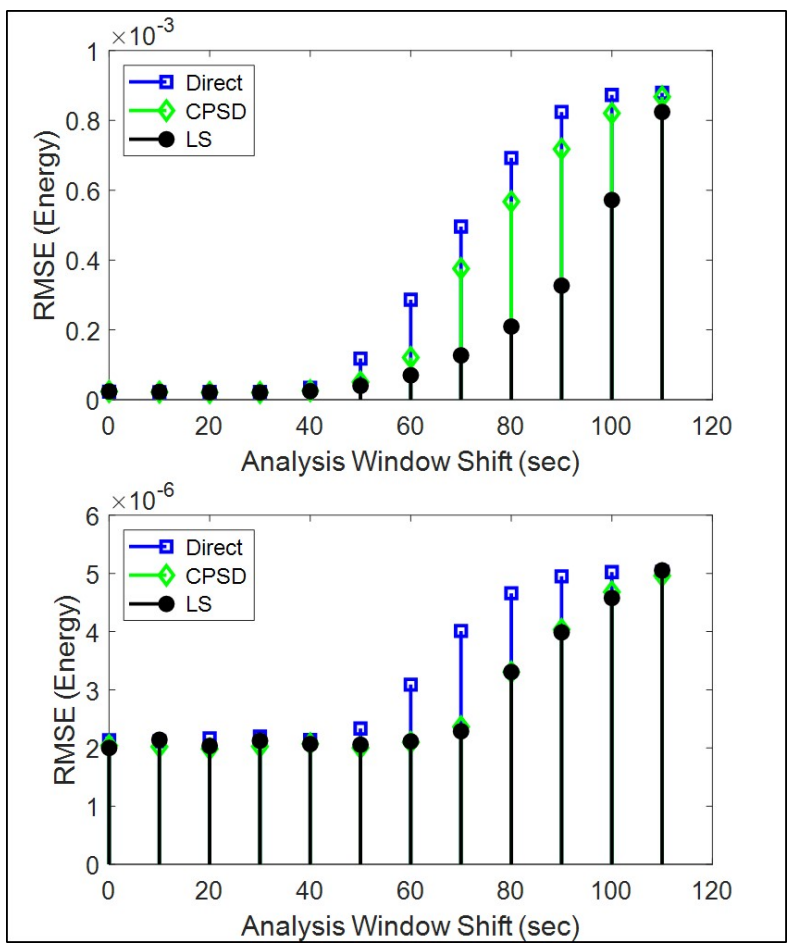

Figure 7. Estimation performance at the source generator for case 1 (top) and 6 (bottom) with shifts in the analysis window.

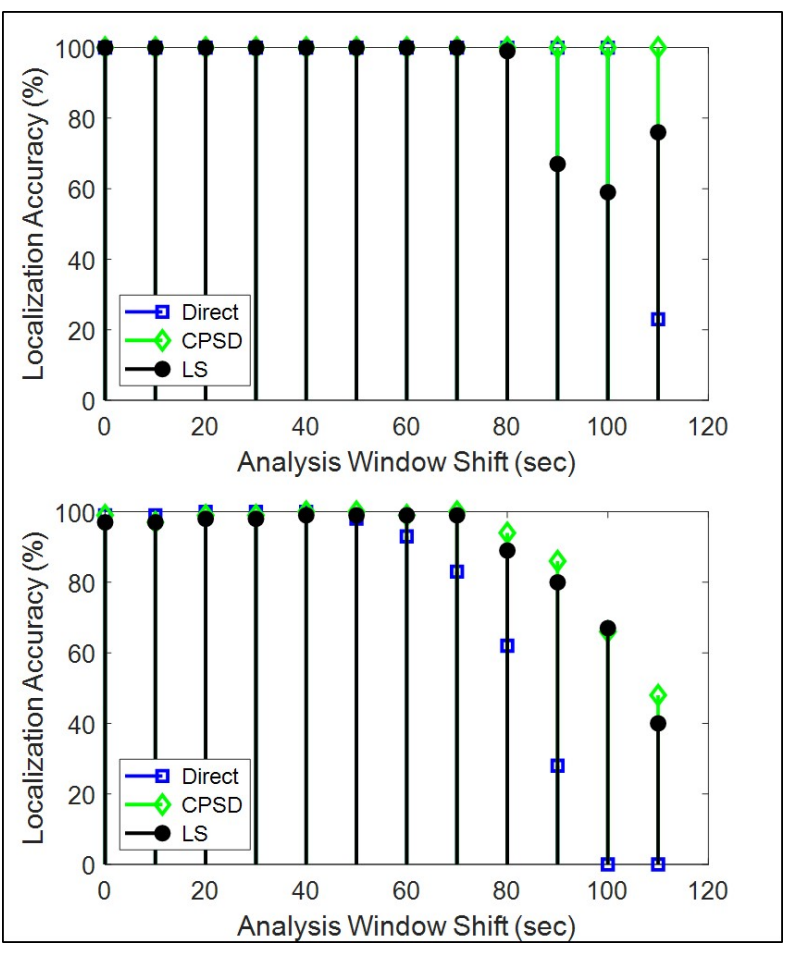

Figure 8. Source localization performance for case 1 (top) and 6 (bottom) with shifts in the analysis window. 


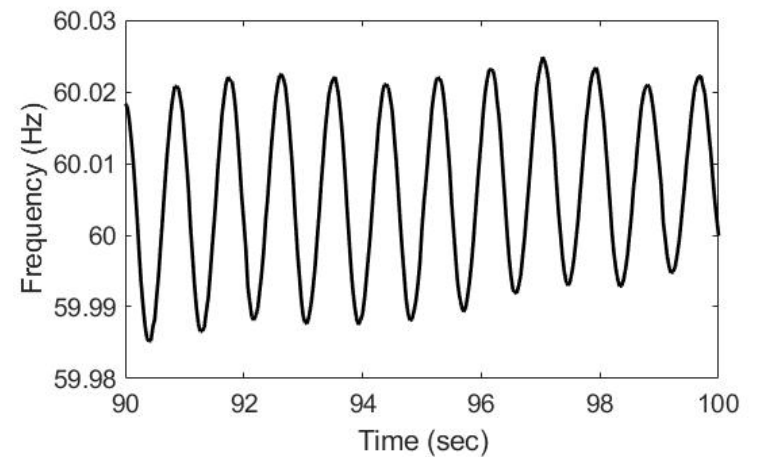

Figure 9. Subset of frequency measurements from the real-world test case.

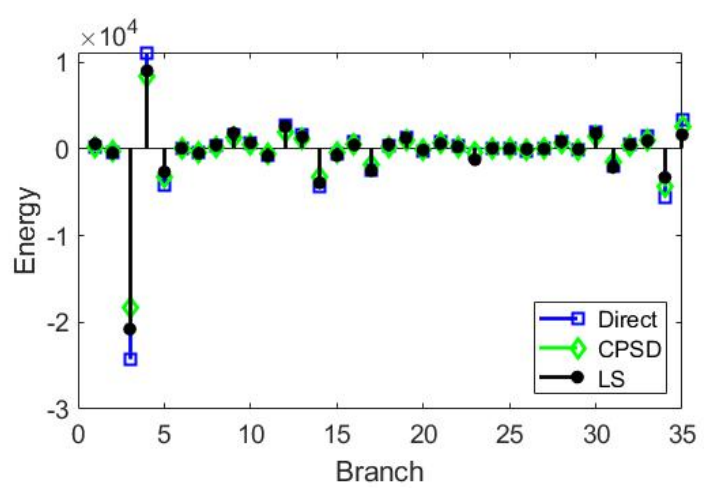

Figure 10. Estimates of the DEF for the real-world test case.

35 branches in the dataset. The branch numbering in Fig. 10 corresponds to the order the signals appear in the dataset downloaded from [9]. From Fig. 10 it is apparent that the DEF is largest on branch 3. The negative value indicates that the oscillation energy is flowing into the substation from the source, which agrees with the information provided in [9].

In Fig. 10, the estimates from each method are in good agreement. Greater disparity was found in test cases two and five, where the oscillations were not as well represented by a sum of sinusoids with constant amplitudes. Even in these cases, all three estimators led to the clear and correct identification of the source. Further discussion of estimator performance is provided in the following section.

\section{Discussion}

The simulation results provide a wealth of information about the performance of the methods proposed in Section 3 and [1]. Under ideal conditions when the oscillation has a constant frequency and amplitude and its parameters are known, each of the methods performs similarly. This is made clear in Fig. 3. Often, real-world conditions can be well represented by this case, but the sensitivity of algorithms under more challenging circumstances is important.

From Fig. 4, it is clear that the Direct method is much more robust than the CPSD and LS methods to errors in the oscillation's frequency. Errors in DEF estimation result in degraded source localization performance, as demonstrated in Fig. 5. Though similar in DEF estimation, the CPSD significantly outperforms the LS method in source localization.

Including a non-integer number of oscillation periods in the analysis window was shown to have a much smaller impact on estimation performance. Though DEF estimates from the CPSD method were degraded, source localization remained unchanged.

The importance of identifying time periods containing sustained oscillations was demonstrated in Figs. 7 and 8 . The Direct method appears to be the most sensitive to errors in selecting the analysis window and the LS method appears to be the least sensitive. This sensitivity may be the least concern because the analysis window is not particularly difficult to select, especially in offline analyses. Significant errors did not occur until large shifts in the analysis window were applied.

Overall, results indicate that all three methods are viable under certain conditions. The Direct method performed reliably, but the required filtering may be disadvantageous in online analyses where the filter design must be automated. The CPSD method is advantageous because it does not require filtering, is very simple to implement, and can analyze multiple harmonics simultaneously. However, its sensitivities may limit it. The DFT method provides estimates identical to those from the CPSD method, but it also estimates the FO's amplitude and phase in each signal. The LS method also provides these estimates, but it is more complex to implement, requires model order selection, and is sensitive to errors in the FO parameters.

With each method possessing advantages and disadvantages, each approach may prove valuable for different applications. The methods can also be used in parallel to provide validation for the results, making the overall implementation more robust.

\section{Conclusion}

Forced oscillations are a significant concern for reliable power system operation. Methods are needed to quickly and accurately identify the oscillation source so that it can be addressed. The previously proposed DEF has been shown to be a reliable indicator of a FO's source. In this paper, a new expression for the DEF 
under commonly observed conditions was developed. Based on this expression, three new estimators for the DEF were derived. The statistical performance of the methods was evaluated using Monte Carlo trials, allowing the sensitivity of each approach under various circumstances to be examined. The new estimators were also validated using real-world measurements. The new methods may be used under circumstances that align with their advantages or deployed alongside the existing method to provide validation.

\section{Appendix}

The approximation in (5) was found empirically. The following offers some insight and support for its use. The initial claim was

$$
\begin{aligned}
& \ln \left(V_{s}+A_{V} \cos \left(\omega_{F O} t+\theta_{V}\right)\right) \\
& \approx \ln \left(V_{s}\right)+\frac{A_{V}}{V_{s}} \cos \left(\omega_{F O} t+\theta_{V}\right)
\end{aligned}
$$

for $V_{s} \gg A_{V}$. The condition $V_{s} \gg A_{V}$ is well justified because even large oscillations are not of comparable size to nominal transmission voltages.

Inverting the natural logarithm on each side of (32),

$$
\begin{aligned}
V_{s}+A_{V} & \cos \left(\omega_{F O} t+\theta_{V}\right) \\
& \approx e^{\ln \left(V_{s}\right)+\frac{A_{V}}{V_{s}} \cos \left(\omega_{F O} t+\theta_{V}\right)} \\
& =V_{s} e^{\frac{A_{V}}{V_{s}} \cos \left(\omega_{F O} t+\theta_{V}\right)} .
\end{aligned}
$$

Dividing both sides by $V_{s}$,

$$
1+\frac{A_{V}}{V_{s}} \cos \left(\omega_{F O} t+\theta_{V}\right) \approx e^{\frac{A_{V}}{V_{s}} \cos \left(\omega_{F O} t+\theta_{V}\right)} .
$$

Noting that the cosine terms are bounded on the interval $[-1,1]$ and that $\frac{A_{V}}{V_{s}}$ approaches zero for $V_{s} \gg A_{V}$ leads to the final result $1 \approx 1$. Thus, the approximation holds for the condition $V_{s} \gg A_{V}$.

\section{References}

[1] S. Maslennikov, B. Wang, and E. Litvinov, "Locating the source of sustained oscillations by using PMU measurements," in 2017 IEEE Power Energy Society General Meeting, pp. 1-5, July 2017.

[2] B. Wang and K. Sun, "Location methods of oscillation sources in power systems: a survey," Journal of Modern Power Systems and Clean Energy, vol. 5, pp. 151-159, Mar 2017.

[3] J. Seppänen, J. Turunen, A. Nikkilä, and L. Haarla, "Resonance of forcing oscillations and inter-area modes in the Nordic power system," in 2018 IEEE PES Innovative Smart Grid Technologies Conference Europe (ISGT-Europe), pp. 1-6, Oct 2018.
[4] S. A. N. Sarmadi and V. Venkatasubramanian, "Inter-area resonance in power systems from forced oscillations," IEEE Transactions on Power Systems, vol. 31, pp. 378-386, Jan 2016.

[5] S. A. Nezam Sarmadi, V. Venkatasubramanian, and A. Salazar, "Analysis of November 29, 2005 Western American oscillation event," IEEE Transactions on Power Systems, vol. 31, pp. 5210-5211, Nov 2016.

[6] "Diagnosing equipment health and mis-operations with PMU data," tech. rep., North American SynchroPhasor Initiative (NASPI), https://www.naspi.org/node/365, 2015.

[7] U. Agrawal, J. W. Pierre, J. Follum, D. Duan, D. Trudnowski, and M. Donnelly, "Locating the source of forced oscillations using PMU measurements and system model information," in 2017 IEEE Power Energy Society General Meeting, pp. 1-5, July 2017.

[8] L. Chen, Y. Min, and W. Hu, "An energy-based method for location of power system oscillation source," IEEE Transactions on Power Systems, vol. 28, pp. 828-836, May 2013.

[9] S. Maslennikov, B. Wang, Q. Zhang, a. Ma, a. Luo, a. Sun, and E. Litvinov, "A test cases library for methods locating the sources of sustained oscillations," in 2016 IEEE Power and Energy Society General Meeting (PESGM), pp. 1-5, July 2016.

[10] R. Xie and D. J. Trudnowski, "Tracking the damping contribution of a power system component under ambient conditions," IEEE Transactions on Power Systems, vol. 33, pp. 1116-1117, Jan 2018.

[11] L. Vanfretti, L. Dosiek, J. Pierre, D. Trudnowski, J. Chow, R. Garcia-Valle, and U. Aliyu, "Application of ambient analysis techniques for the estimation of electromechanical oscillations from measured PMU data in four different power systems," Electrical Power, European Transactions on, vol. 1, pp. 1640-1656, October 2011.

[12] J. Follum and J. W. Pierre, "Detection of periodic forced oscillations in power systems," IEEE Transactions on Power Systems, vol. 31, pp. 2423-2433, May 2016.

[13] P. Stoica and R. L. Moses, Spectral Analysis of Signals. Pearson/Prentice Hall Upper Saddle River, NJ, 2005.

[14] J. Follum, J. W. Pierre, and R. Martin, "Simultaneous estimation of electromechanical modes and forced oscillations," IEEE Transactions on Power Systems, vol. 32, pp. 3958-3967, Sep. 2017.

[15] D. Trudnowski, D. Kosterev, and J. Undrill, "PDCI damping control analysis for the western North American power system," in Power and Energy Society General Meeting (PES), 2013 IEEE, pp. 1-5, July 2013.

[16] P. B. Reddy and I. A. Hiskens, "Limit-induced stable limit cycles in power systems," in 2005 IEEE Russia Power Tech, pp. 1-5, June 2005.

[17] S. Kay, Fundamentals of Statistical Signal Processing, Volume I: Estimation Theory. Prentice Hall, 1993.

[18] B. Porat, A Course in Digital Signal Processing, vol. 1. Wiley New York, 1997.

[19] J. Follum and J. Pierre, "Time-localization of forced oscillations in power systems," in 2015 IEEE Power Energy Society General Meeting, pp. 1-5, July 2015. 